\title{
Editorial
}

\section{Possible Impact of COVID 19 on Health Care Professionals}

\author{
Butchi Raju Akondi ${ }^{*}$, Amit Vanka ${ }^{2}$ and Shanthi Vanka ${ }^{2}$ \\ 'Departmnet of Clinical Pharmacy and Pharmacology, Ibn Sina National College for Medical Studies, \\ Jeddah, Saudi Arabia; drraju2020@gmail.com \\ ${ }^{2}$ Departmnet of Preventive Dentistry, Ibn Sina National College for Medical Studies, Jeddah, Saudi Arabia
}

A novel coronavirus is the source of a major public health problem of global magnitude. The pandemic started from Wuhan, China, wherein the Chinese center for disease control and prevention officially identified novel coronavirus as the causative pathogen of COVID-19. As of May 13, 2020, a total of 41,31,794 individuals are affected worldwide, resulting in 2,85,328 deaths. ${ }^{2}$ The mode of spread is not fully understood, but the most probable route for the spread includes: respiratory droplets during coughing or sneezing, aerosol with concerns about airborne spread as well. As there is no availability of effective drugs or vaccines, infection prevention and control strategies to prevent or limit transmission of COVID-19 is the only possible recourse.

Health professionals (HP) are in the front line of the response to COVID-19 outbreak and the pandemic is stretching health systems worldwide. The rapidly increasing demand on health facilities and health care workers threatens to overstretch and even collapse the existing healthcare systems.

Doctors, nurses, Pharmacists and other health care professionals have an important role to play in identifying, reporting and managing potential cases of COVID-19. A multitude of hazards are faced by the HP including: increased risk of exposure to the pathogen, stressful working conditions and psychological distress. ${ }^{2} \mathrm{HP}$ are often faced with a need to provide emotional support to patients isolated from their families. The dilemma regarding how to align their desire and duty to patients with those to family and friends, and how to provide care for all severely affected individuals together puts tremendous burden on the HP.

The dental fraternity is at a high risk of cross infection due to the nature of procedures performed in the dental settings. Most dental procedures lead to aerosol and splatter generation and general consensus appears to be that most elective procedures may be rescheduled and emergency treatment be executed under the strictest infection control. ${ }^{3,4}$ Another reason cited for delaying dental procedures stems from the shortage of personal protection equipment (PPE) in some parts of the world which has been diverted to the more pressing needs in hospitals and emergency care settings.

Often, communities may have first point contact with pharmacists, since they are the most approachable healthcare providers within the system. ${ }^{5}$ Pharmacists associated with hospitals, clinics and community settings are well trained to manage patients with infectious diseases and can significantly contribute to the healthcare system, even more-so during the current crisis, wherein the burden on health care system is tremendous. Reliable information and advice may be provided by the pharmacists with COVID-19 related concerns, including proper referrals as the situation demands. In order to efficiently provide care to patients under the current circumstances, a few guidelines are worth mentioning. ${ }^{6,7}$

${ }^{*}$ Author for correspondence 


\section{Adherence to Established Occupational Safety and Health Procedures}

Infection-control constitutes the key measure to be adopted in the strictest sense in order to contain the spread of infection. Regular attendance in safety and health training measures being provided at respective institutions are crucial as is continually updating oneself with the regulations from government and regulatory bodies. Additionally, hand hygiene measures and surface decontamination measures for mobile phones, car keys, elevators, and chair are all essential to prevent contamination and spread of virus at the workplace.

\section{Moral Issues}

The risk of infection spread amongst the subgroup of $\mathrm{HP}$ is quite high. Hence it is imperative that healthcare providers should self-monitor for signs of illness. Reporting to management in the event of; experiencing physical signs of illness, undue mental stress and any such challenges, in order to provide a reasonable justification of imminent danger to health or life, is a crucial element of providing safety for the patients and themselves.

\section{Family or Community Spread}

The danger of spreading the virus amongst family members or community is a real one for healthcare providers. Several managements have allocated separate living quarters for healthcare providers who are in regular contact with infected individuals. In the event that healthcare providers come in regular contact with the family or community, it is important that a regular check be kept on their health status as well and infection control measures such as change of clothing at workplace may be encouraged.

\section{Team Work and Leadership}

The workload amongst the healthcare professionals at present is unevenly distributed. Dentists, pharmacists and can be called upon to play supplementary roles in this crisis in order to reduce the burden on the physicians and nurses who are being stretched to the limits. The pharmacy and dental councils and concerned organizations should play a vital role in framing the guidelines for greater involvement of these HP's. Healthcare professionals should receive adequate moral and psychological support as well in the form of regular counseling sessions.

\section{References}

1. Zhu N, Zhang D, Wang W, et al. A Novel Coronavirus from Patients with Pneumonia in China, 2019. N Engl J Med. 2020; 382(8):727-733.

2. Covid19.who.int. 2020. WHO Coronavirus Disease (COVID-19) Dashboard. [online] Available at: https:// covid19.who.int/ [Accessed 10 May 2020].

3. Coulthard P. Dentistry and coronavirus (COVID-19)moral decision-making. British Dental Journal. 2020; 228(7):503-5.

4. Meng L, Hua F, Bian Z. Coronavirus disease 2019 (COVID19): emerging and future challenges for dental and oral medicine. Journal of Dental Research. 2020; 99(5):481-7.

5. Cadogan CA, Hughes CM. On the frontline against COVID19: Community pharmacists' contribution during a public health crisis [published online ahead of print, 2020 Mar 31]. Res Social Adm Pharm. 2020; S1551-7411(20)30292-8.

6. World health organization. 2020. WHO Guidance for Health Workers. [online] Available at: https://www.who. int/emergencies/diseases/novel-coronavirus-2019/technical-guidance/health-workers [Accessed 10 May 2020].

7. Adams, J. and Walls, R., 2020. Supporting the Health Care Workforce during the COVID-19 Global Epidemic. JAMA, 323(15), p. 1439. 